\title{
SOME MULTIPLE INTEGRAL INEQUALITIES VIA THE DIVERGENCE THEOREM
}

\section{SILVESTRU SEVER DRAGOMIR}

Abstract. In this paper, by the use of the divergence theorem, we establish some inequalities for functions defined on closed and bounded subsets of the Euclidean space $\mathbb{R}^{n}, n \geqslant 2$.

Mathematics subject classification (2010): 26D15.

Keywords and phrases: Multiple integral inequalities, divergence theorem, Green identity, GaussOstrogradsky identity.

\section{REFERENCES}

[1] H. Budak AND M. Z. SARIKAYa, Generalized weighted Čebysev and Ostrowski type inequalities for double integrals, TWMS J. Appl. Eng. Math. 7 (2017), no. 2, 272-281.

[2] H. BUdAK AND M. Z. SARIKAYA, On weighted Grüss type inequalities for double integrals, Commun. Fac. Sci. Univ. Ank. Sér. A1 Math. Stat., 66 (2017), no. 2, 53-61.

[3] S. S. DRAGOMIR, Double integral inequalities of Hermite-Hadamard type for h-convex functions on linear spaces, Analysis (Berlin) 37 (2017), no. 1, 13-22.

[4] S. S. DRAGOMIR, Some inequalities for double and path integrals on general domains via Green's identity, Preprint RGMIA Res. Rep. Coll. 22 (2019), Art. 55, 17 pp. Online http://rgmia.org/papers/v22/v22a55.pdf.

[5] S. S. DRAGOMIR, Some triple integral inequalities for functions defined on 3-dimensional bodies via Gauss-Ostrogradsky identity, Preprint RGMIA Res. Rep. Coll. 22 (2019), Art. 60, 21 pp. Online http://rgmia.org/papers/v22/v22a60.pdf.

[6] B. G. PachPatte, New inequalities of Ostrowski and Grüss type for triple integrals, Tamkang J. Math. 40 (2009), no. 2, 117-127.

[7] D. B. PachPatte, Some Ostrowski type inequalities for double integrals on time scales, Acta Appl. Math. 161 (2019), 1-11.

[8] M. SINGER, The divergence theorem, Online https://www.maths.ed.ac.uk/ ${ }^{j m f / T e a c h i n g / L e c t u r e s / d i v t h m . p d f . ~}$

[9] W. T. Sulaiman, Integral inequalities concerning triple integrals, J. Concr. Appl. Math. 8 (2010), no. 4, 585-593. 